and the DSM-IV/ICD-11 revisions are discussed by Regier et al.

Gershon offers a historical picture of behavior genetics and puts this in the perspective of the hunt for genes for other complex disorders. He provides a balanced discussion of the possibilities for use and misuse of genotype data as for heritable traits such as intelligence and alcoholism.
The third part of the book focuses on neurobiology and mood disorders. Drevets offers a comprehensive chapter on brain structural abnormalities in mood disorders and discusses the implications for the pathogenesis of emotion dysregulation based on PET, MRI and postmortem brain studies.

The two final chapters are devoted to educational trends in psychiatric training. The first chapter, by Rubin and Zorumski, focuses on workforce issues and advances in neuroscience. The second, by Hudziak argues that advances in genomics and neuroscience lay the foundation for an educational model that will increase the likelihood that more science-minded medical students will choose careers in psychiatry.

\title{
Schizophrenia: Challenging the Orthodox
}

\author{
Editors: Colm McDonald, Katja Schulze, Robin M. Murray, and Padraig Wright
}

(2004). Taylor \& Francis, 22 I pp, US\$69.95, ISBN I84I843776.

Schizophrenia: Challenging the Orthodox, edited by Colm McDonald, Katja Schulze, Robin Murray and Padraig Wright, offers a more in-depth review of the disorder that was once described as 'the heartland of psychiatry' and this book as 'the Holy Grail' of gene searching. This book also follows from a conference meeting (the inaugural meeting of the European Foundation for Psychiatry) and was recommended for British Medical Association Awards 2005. It consists of 23 short chapters, written by a large number of authors and is divided into sections on Brain Imaging; Genetic Research; Epidemiology; Prodrome and Early Intervention; Psychopharmacology and Psychology; Cognitive
Therapy; and Schizophrenia and Bipolar Disorder.

The section on genetics offers an overview of important linkage and association findings and the encouraging story from Iceland about the discovery of neuregulin as a major susceptibility gene for schizophrenia through standard operational protocols for positional cloning. The section on epidemiology looks at possible risk (e.g., urbanicity) and protective (e.g., cigarette smoking) factors of schizophrenia. One of the chapters by Murray et al. is devoted to the increased risk of schizophrenia in methamphetamine and cannabis users, suggesting that (part of) the increased risk may reflect causality. The role of 'pot' is also considered by
D'Souza in the section on pharmacology and psychology. Other chapters in this section cover the role of dopamine system. The book concludes with an informative series of papers on the - by now accepted - comorbidity of schizophrenia and bipolar disorder in which shared genetic architecture, shared brain characteristics from imaging studies and developmental trajectories are examined.

All three books are very accessible. Twin researchers may enjoy the first book the most, but all three have much to offer to anyone interested in psychiatric genetic research.

\footnotetext{
Dorret I. Boomsma

Department of Biological Psychology Vrije Universiteit, Amsterdam,

the Netherlands
} 